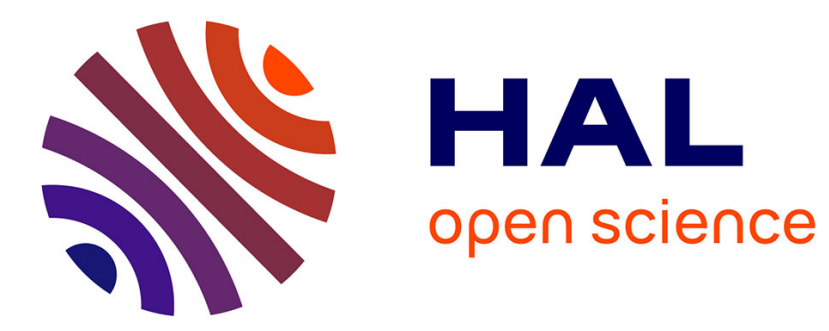

\title{
Tridentate complexes of group 4 bearing bis-aryloxide N-heterocyclic carbene ligand: Structure, spin density and charge states
}

Sébastien Le Roux, Guido Ori, Stéphane Bellemin-Laponnaz, Mauro Boero

\section{- To cite this version:}

Sébastien Le Roux, Guido Ori, Stéphane Bellemin-Laponnaz, Mauro Boero. Tridentate complexes of group 4 bearing bis-aryloxide N-heterocyclic carbene ligand: Structure, spin density and charge states. Chemical Physics Letters, 2021, 781, pp.138888. 10.1016/j.cplett.2021.138888 . hal-03323181

\section{HAL Id: hal-03323181 \\ https://hal.science/hal-03323181}

Submitted on 20 Aug 2021

HAL is a multi-disciplinary open access archive for the deposit and dissemination of scientific research documents, whether they are published or not. The documents may come from teaching and research institutions in France or abroad, or from public or private research centers.
L'archive ouverte pluridisciplinaire HAL, est destinée au dépôt et à la diffusion de documents scientifiques de niveau recherche, publiés ou non, émanant des établissements d'enseignement et de recherche français ou étrangers, des laboratoires publics ou privés. 


\title{
Tridentate Complexes of Group 4 Bearing Bis-Aryloxide $\mathrm{N}-\mathrm{Heterocyclic} \mathrm{Carbene} \mathrm{Ligand:} \mathrm{Structure,} \mathrm{Spin} \mathrm{Density}$ and Charge States
}

\author{
Sébastien Le Roux, Guido Ori, Stéphane Bellemin-Laponaz, Mauro Boero \\ 5 University of Strasbourg, Institut de Physique et Chimie des Matériaux de Strasbourg, CNRS, UMR 7504, \\ 23 rue du Loess, F-67034 France
}

\begin{abstract}
Metal carbene complexes represent an ubiquitous class of compounds in organomettalic chemistry able to trigger a wealth of catalytic reactions of both fundamental and industrial processes. By resorting to first principles approaches, we focus on the fundamental features of these complexes in which the metal center can be either Hf, Ti or Zr. These specific systems show interesting features in their neutral and charged $(+1)$ states. Yet, we provide evidence of the fact that the nature of the metallic ion does not have a significant impact on the structure of the ligand. Conversely, the removal of one electron (charge state +1 ) induces a perturbation of the ligand conformation and this, in turn, is prone to affect the catalytic properties of the complex, carrying the singly occupied electronic level (spin distribution of the system) delocalized over the carbene ligand.
\end{abstract}

\section{Introduction}

$\mathrm{N}$-heterocyclic carbenes (NHCs) are currently widely used ligands in a variety of 10 organometallic chemistry reactions, ranging from homogeneous catalysis and materials science to biomedical applications.[1, 2, 3, 4, 5, 6, 7, 8] NHCs ligands consist of cyclic ring-shaped carbon-based moieties containing as a minimal building block a carbene group connected directly to at least one nitrogen atom within the heterocycle. Since the seminal work in the late 60's of Wanzlick and Öfele, $[9,10]$ and the subsequent break-

15 throughs by Bertrand and Arduengo,[11, 12] the interest in coordination chemistry of NHCs underwent a considerable increase in view of the potential applications that could be disclosed by changing and tuning the transition metal (TM) center. However, results concerning the use of oxophilic and electropositive early transition metal species supported by NHC carbene have been much less investigated as opposed to the rich lit20 erature on late transition metal NHC complexes. [7, 6, 13, 14] This is mainly due to the difficulty in synthesizing complexes sufficiently stable to be manipulated and used in catalytic reactions without giving rise to dissociation or disassembling of the NHC system. As a consequence, also quantum chemical investigations into this type of systems have been so far rather limited.[15, 16, 17, 18] NHC carbene contains a nonbonded 25 singlet lone pair and is an electron-rich neutral donor ligand, primarily acting as exceptionally strong $\sigma$-donating L-type ligands. The nucleophilic ability of this type of carbene is associated with both the $\sigma$-electron-withdrawing and $\pi$-electron-donating character of the nitrogen centers. Due to their strong $\sigma$-electron-donating properties,

Email address: sebastien. leroux@ipcms. unistra.fr (Sébastien Le Roux) Preprint submitted to Elsevier 
NHC ligands form stronger bonds with transition metal (TM) centers than most classical ligands, such as phosphines, thus giving TM complexes that are highly versatile (i.e. adaptable $\pi$-accepting abilities) and generally resistant to decomposition.[19] Additionally, the empty $p$-orbital at the carbene carbon atom is poised to accept $\pi$-electron

5 density from a TM (i.e., $\pi$-back-bonding). However, due to competing $\pi$-overlap of electron-rich nitrogen atoms adjacent to the carbene atom, the extent to which NHCs are capable of participating in this type of bonding interaction has been under debate and it is of particular interest.[20] Among the NHCs ligands documented so far, NHCincorporating pincer-type chelating tridentate ligands is receiving increasing attention

to thanks to their chemical robustness, stability and versatility in catalytic performance. $[21,22,23,19,14]$ In particular, the bis-aryloxide N-heterocyclic carbene tridentate ligand ${ }^{t \mathrm{Bu}}(\mathrm{OCO})^{2-}$ has been shown to be suitable to host early transition metals, with a high versatility ranging from group 3 to group 7 TM.[21] The ${ }^{t \mathrm{Bu}}(\mathrm{OCO})^{2-}$ ligand features a central NHC donor flanked on each side by an ortho- and para-substituted ${ }_{5}$ phenolate moiety in which the $t \mathrm{Bu}$ groups provide a high degree of steric protection that allows an unprecedented ability in coordinating $\mathrm{Ti}, \mathrm{Zr}$ and $\mathrm{Hf}$ in a very stable way, thus providing highly robust $\left[{ }^{t \mathrm{Bu}}(\mathrm{OCO})\right] \mathrm{MR}_{2,3}$ (with $\mathrm{R}=\mathrm{Cl}, \mathrm{THF}, \mathrm{O}^{i} \mathrm{Pr}$ ) complexes displaying exceptional catalytic performances. [23, 24, 25] The relative high degree of robustness and steric protection of this NHC-incorporating pincer-type chelating

20 ligand is also enhanced by the direct connection between the nitrogen atoms of the central NHC and the two phenolate moieties. This ligand contains two sterically hindered phenolate groups that can become redox-active.[26] In particular, it has been shown that such a redox active ligand is able to support TM complexes in four different oxidation states, thus conferring a high degree of both flexibility and stability 25 to the system.[27, 28] In addition, transition metal complexes containing non-innocent ligands such as redox active phenolates arouses great interest due to their interest in fields such as bioninorganic chemistry or catalysis.[29, 30]

Since the ${ }^{t \mathrm{Bu}}(\mathrm{OCO})^{2-}$ ligand displays a remarkable ability in stabilizing $\mathrm{d}^{0}$ group 4 metals, specifically $\mathrm{Ti}, \mathrm{Zr}$, and $\mathrm{Hf}$, and being the ligand redox active, in the present so work we undertook a thorough theoretical investigation of these complexes to shed some light on their properties at an atomic-level. By resorting to first principles simulations, within the density functional theory framework, we present an analysis of the electronic and structural properties of $\mathrm{d}^{0}$ complexes $\left[{ }^{t \mathrm{Bu}}(\mathrm{OCO})\right] \mathrm{MCl}_{2}(\mathrm{THF})(\mathrm{M}=\mathrm{Ti}$, $\mathrm{Zr}$, Hf) in their neutral form and in the case in which these same systems undergo a

35 typical oxidation process resulting in the lack of one electron (i.e. cationic form). This confers both an overall charge and a related distribution of the spin density on the complex that, in turn, can influence the chemical and physical properties of the system with consequences on the catalytic activity.

Only a few theoretical studies on the orbital interaction of $\mathrm{M}-\mathrm{C}$ (NHC) bonds of early

${ }_{40}$ transition metal complexes of metals in their higher or highest oxidation state (electronic configuration $\mathrm{d}^{0}-\mathrm{d}^{2}$ ) are currently available. [31, 32, 33, 34]

\section{Simulated Model and Theoretical Methods}

In this work we make a comparative analysis of the stability, atomic structure and 45 and electronic properties of the NHC complexes carrying different TMs in their neutral (singlet state) and charged (+1, doublet state) charged states. [35] We recall that within any standard DFT approach, the total charge of the system is unambiguously defined by the sum of the ionic point-like charges minus the integral of the electron 
density $\rho(\mathbf{x})$. As such, unless projections or charge partitions methods as the ones used in the reminder of this work are adopted, it is difficult to attribute a net charge to a specific atom. We constructed $\left[{ }^{t \mathrm{Bu}}(\mathrm{OCO})\right] \mathrm{MCl}_{2}$ (THF) complexes made of 95 atoms containing one metal center $M$ in which the $\mathrm{TM}$ is either $\mathrm{Ti}(Z=22), \mathrm{Zr}(Z=40) \mathrm{Hf}$

$5 \quad(Z=72)$. In these systems, the TM coordinates two $\mathrm{Cl}$ atoms, one tridentate carbene ligand $\left({ }^{t \mathrm{Bu}}(\mathrm{OCO}),\left(\mathrm{C}_{31} \mathrm{H}_{44} \mathrm{O}_{2} \mathrm{~N}_{2}\right)\right)$, and one tetrahydrofuran $(\mathrm{THF})$ molecule $\left(\mathrm{C}_{4} \mathrm{H}_{8} \mathrm{O}\right)$. Because of its high air stability[22, 23, 25] we have selected $\left[{ }^{t \mathrm{Bu}}(\mathrm{OCO})\right] \mathrm{MCl}_{2}$ (THF) complexes where $\mathrm{M}$ is 6 fold coordinated to 1 solvent THF molecule, 2 chlorine atoms and 1 bis-aryloxide $n$-heterocyclic carbene ligand. This complex can be obtained in ${ }_{10}$ quantitative yield and fully characterized (single crystal X-ray crystallography, ${ }^{1} \mathrm{H}$ and ${ }^{13} \mathrm{C}$ NMR and elemental analysis). [22, 23, 25] On the contrary, the 5 fold coordinated metal complex $\left[{ }^{t \mathrm{Bu}}(\mathrm{OCO})\right] \mathrm{MCl}_{2}$, that does not bear the solvent molecule and that can only be synthesized by using a noncoordinative solvent (such as $\mathrm{CH}_{2} \mathrm{Cl}_{2}$ and toluene), has been found highly air-sensitive and therefore less stable.

15 Our computational approach is base on the spin-unrestricted density functional theory (DFT) in which the generalized gradient approximation (GGA) due to Becke (B) is adopted to describe the exchange interaction, whereas a Lee, Yang and Parr (LYP) functional accounts for the correlation. [36, 37] Valence electrons are treated explicitly and expanded on a plane wave (PW) basis set with an energy cutoff of 70 Ry for both

20 Hf and $\mathrm{Zr}$ and $80 \mathrm{Ry}$ for Ti. The Brillouin zone sampling is limited to the $\Gamma$ point. The sightly higher cutoff used for $\mathrm{Ti}$ is justified by its electronic structure requiring a finer mesh in real space for a good accuracy, thus translating into a larger cutoff in the Fourier space. For Zr, semicore states turned out to be necessary in the construction of the pseudopotential (PP) to model the core-valence interaction. [38] The valence-core

25 interaction is described by norm conserving Trouiller-Martins pseudopotentials [39] and the construction of the pseudopotentials was done by using the atomic configurations [Ar] $3 d^{2} 4 s^{2}$ for Ti, [Ar] $3 d^{10} 4 p^{6}$ ] $4 s^{2} 4 d^{2} 5 s^{2}$ for $\mathrm{Zr}$ and [Xe] $4 f^{14} 5 d^{2} 6 s^{2}$ for $\mathrm{Hf}$, the parts in square bracket being the core parts. The calculations were performed in simulations cells of size $15 \times 15 \times 25 \AA^{3}$ beyond which the periodic boundary condiso tions were released according to the Barnett-Landman scheme[40] for isolated systems thus eliminating spirituous effects induced by periodically repeated images generally biasing in PWs approaches. Furthermore to consider dispersive forces and reproduce as accurately as possible the electronic structure of the organometallic complexes, [41, 42] semi-empirical van der Waals interactions were included in the calculation and treated 5 according to the Grimme D2 formulation. [43] The initial conformations used to construct the models were obtained from the partial X-ray crystallographic coordinates provided in Refs. [22, 23, 25] The geometry of each system was fully optimized in two different steps. In the first one, a geometry relaxation was performed by standard forces minimization via direct inversion in the iterative subspace,[44] and then, ${ }_{0}$ in the second step, a damped molecular molecular dynamics[45] was performed applying a friction force to the atoms (atoms velocities scaled by a factor 0.95 at each step) to allow the system to attain a stable minimum. In all dynamical simulations, the Car-Parrinello[46, 47] method (CPMD) was adopted, allowing for a simultaneous evolution of both the electronic and ionic degrees of freedom in a self-consistent varia45 tional procedure. A fictitious electron mass of 500 a.u. (i.e. in units of $m_{e} a_{0}^{2}$ where $m_{e}$ is the electron mass and $a_{0}$ is the Bohr radius) and a time step of $\Delta t=5$ a.u. $(\simeq 0.12 \mathrm{fs})$ were used for the numerical integration of the equations of motion. The electronic structure of the organometallic complexes was then quantified in terms of Kohn-Sham (KS) orbitals, with special attention on the states around the HOMO-LUMO gap. This ${ }_{50}$ analysis was complemented by the calculations of the Bader charges[48, 49] and spin 
density distribution as specified in the following sections, whenever needed to support the discussion.

\section{Results and Discussion}

\subsection{Structural properties}

To assess the relative stability of $\left[{ }^{t \mathrm{Bu}}(\mathrm{OCO})\right] \mathrm{MCl}_{2}$ (THF) complexes, we first determined the role of the conformational orientation of tetrahydrofuran (THF) molecule with respect to the TM center. The initial atomic positions of the $\mathrm{Cl}$ ions and the conformation of the NHC ligand were selected on the basis of the available partial X-ray crystallographic coordinates.[23] We started our simulations using two distinct initial conformations illustrated in Figure 1.

The difference between these two geometries is a rotation by a $90^{\circ}$ of the THF moiety. For each of the $3 \mathrm{TMs}$ (Hf, Ti and $\mathrm{Zr}$ ) a geometry optimization[44] we performed as specified in the former paragraph. Then a damped CPMD dynamics starting from

15 these relaxed structures allowed to better assess the stability of the various conformations. This same simulation protocol was applied to both the neutral and the charged $(+1)$ systems. Independently of the metallic center and charge state, in terms of total energy our calculations show that the most stable isomer is the conformation indicated as a) in Figure 1. Concerning the THF moiety, we observed that the most stable con${ }_{20}$ formation is the one in which the THF has its heterocyclic ring nearly parallel to the $\mathrm{O}_{1}-M-\mathrm{O}_{2}$ axis (Figure 2-left).

Being this conformation the one found at the end of each optimization procedure, hereafter we shall focus only on this a) structure. We remark that for the charged systems, 25 and on the time scale of the CPMD simulations ( $\sim 1.6 \mathrm{ps})$, the conformational rotation of the THF molecule does not occur spontaneously. However, the total energy of the system remains significantly lower, with an energy difference $\left(\Delta_{\mathbf{a}-\mathbf{b}}=-1.36 \mathrm{eV}\right)$ for conformation a) with respect to $\mathbf{b}$ ) all along the optimization and the damped dynamics. On an stability standpoint, the removal of one electron does not perturb significantly so the $\left[{ }^{t \mathrm{Bu}}(\mathrm{OCO})\right] \mathrm{MCl}_{2}$ (THF) complexes, the total energy being just slightly higher (see Table 6), and this holds for all the systems considered in this work.

A general feature to be remarked is the nearly negligible modifications the geometry of the systems undergoes when comparing the neutral and the charged complexes. A detailed analysis of the geometry with the TM site in the octahedral conformation (Fig${ }_{35}$ ure 2-left) is reported in Tables 1 and 2.

The carbene-metal C-TM bond distances obtained by simulation show good agreement with the range of distances found experimentally within the statistical uncertainty affecting our calculations and the experimental measurements (Table 1). These values

40 fairly also fall within the range of C-TM bond distances found for group $4 \mathrm{NHC}$ complexes thus far X-ray characterized according to the CCDC database recently reported

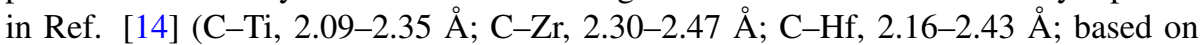
a total of 137 structures).[19] The only noticeable difference with respect of the experimental data is the slightly longer bond distance of the TM with the oxygen of the

${ }_{45}$ THF molecule, $\mathrm{d}_{\mathrm{M}-\mathrm{O}_{m}}$, for all the three metals. We can also observe that $\mathrm{d}_{\mathrm{M}-\mathrm{O}_{m}}$ slightly decreases for the charged systems with respect to the neutral state. Analogously, the distances between the $\mathrm{TM}$ and the $\mathrm{Cl}$ atoms decrease, whereas the separation between 
a)

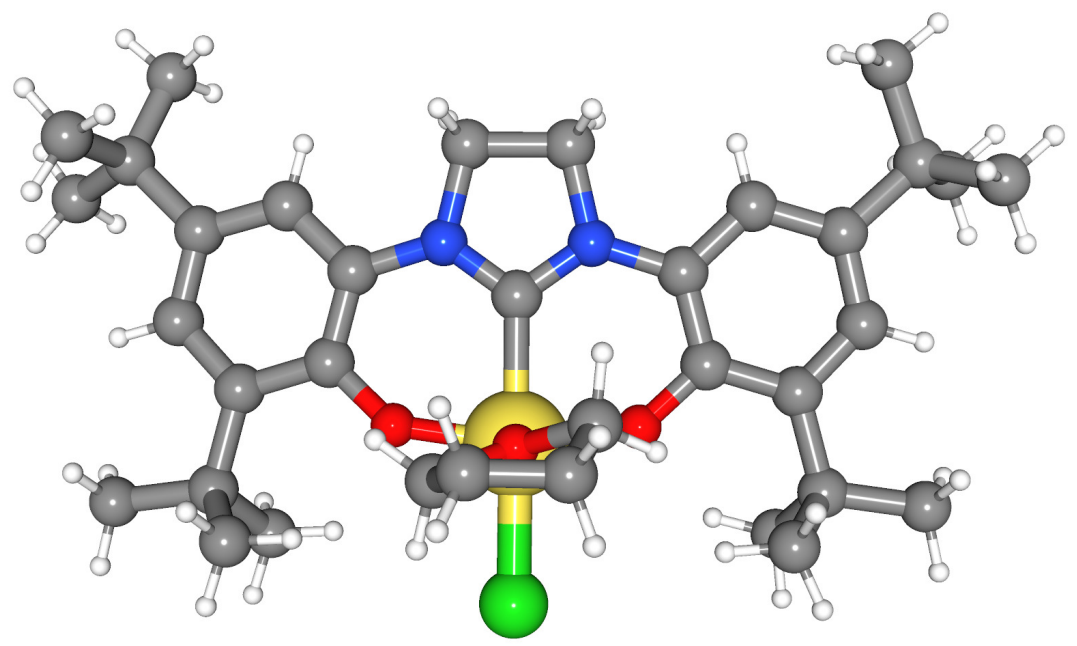

b)

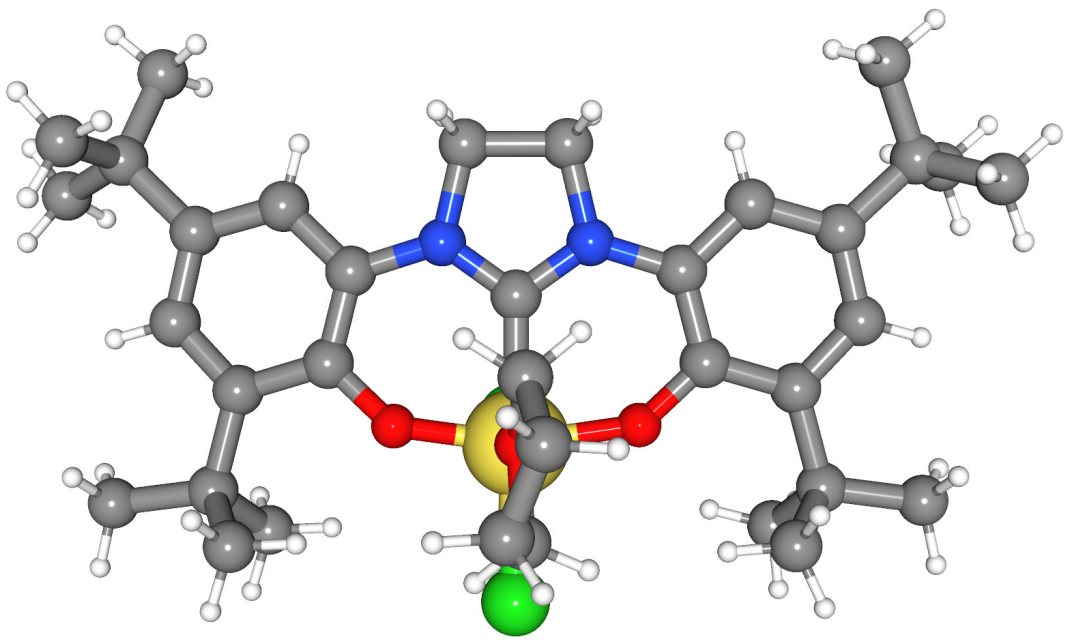

Figure 1: (Color online) Schematic representation of the initial models of our simulations with the 2 conformations of the tetrahydrofuran (THF). Top a) the THF molecule is almost parallel to the $\mathrm{O}_{1}-M-\mathrm{O}_{2}$ axis, bottom b) the THF molecule is tilted by $90^{\circ}$ and its cycle is almost parallel to the $\mathrm{Cl}_{1}-M-\mathrm{Cl}_{2}$ axis (see Figure 2 for details regarding the atomic labels).

the TM and the $\mathrm{O}$ sites of the carbene ligand increase in the charged system. Conversely, no significant changes were observed in the distance between the metal center and the $\mathrm{C}$ atom of the carbene ligand, which turns out to be insensitive to the charge state of the complex. By inspecting the bond angles analysis reported in Table 2, our 5 calculations confirm that the metal atoms exhibit a slightly distorted octahedral geometry as a result of the mer-coordination of the tridentate NHC ligand with O-M-O bite angles of $\sim 162^{\circ}, \sim 153^{\circ}$, and $\sim 156^{\circ}$ for $\mathrm{Ti}, \mathrm{Zr}$ and Hf complexes, respectively. These values fairly agree with the ones obtained by X-ray crystallography $\left(\sim 159^{\circ}, \sim 153^{\circ}\right.$, and $\sim 155^{\circ}$ ). The depletion of an electron in the cationic complexes does not affect 

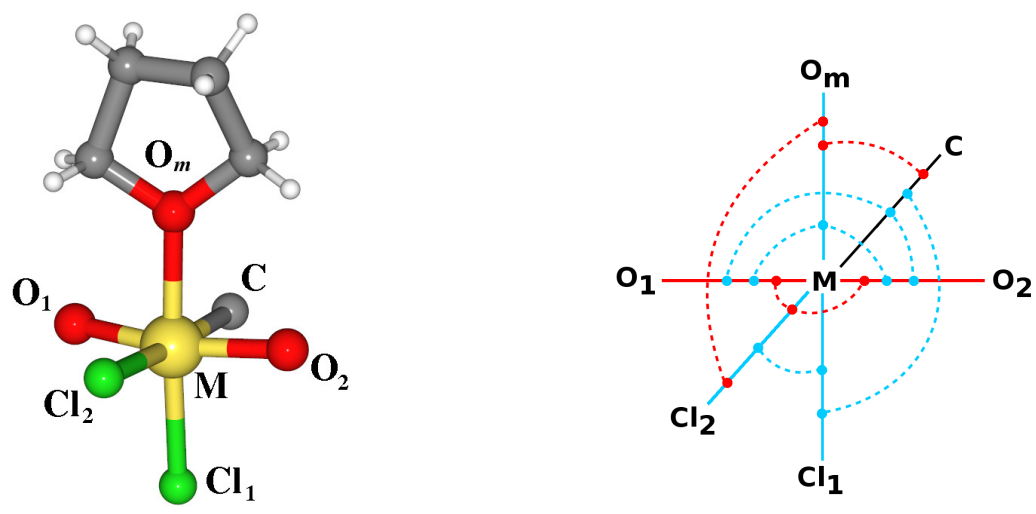

Figure 2: (Color online) Left: schematic of the metallic site in the octahedral conformation. Right: visual overview of the geometry changes in the octahedral site between the neutral and the charged $\left[{ }^{t \mathrm{Bu}}(\mathrm{OCO})\right] \mathrm{MCl}_{2}$ (THF) complex. Red line $(-)=$ bond increase, blue line $(-)=$ bond decrease, red dashed line between points $(\circ---\circ)=$ angle increase and blue dashed line between points $(\circ---\circ)=$ angle decrease.

Table 1: Interatomic distances $(\AA)$ between the metal atoms and their nearest neighbors. For each metal the columns read like: left= neutral system and right= charged system (see Figure 2 for details regarding the atomic labels). The uncertainty of the simulated values is $\pm 0.04 \AA$. The experimental values obtained by X-ray crystallography analysis are reported in parenthesis and taken from Refs. [16, 17, 18] for Ti, Zr, and Hf complexes, respectively.

\begin{tabular}{lrrrrrr}
\hline \hline & \multicolumn{2}{c}{$\mathrm{Ti}$} & \multicolumn{2}{c}{$\mathrm{Zr}$} & \multicolumn{2}{c}{ Hf } \\
& \multicolumn{1}{c}{0} & +1 & 0 & +1 & 0 & +1 \\
\hline $\mathrm{d}_{\mathrm{M}-\mathrm{O}_{m}}$ & $2.29(2.126(2))$ & 2.24 & $2.47(2.279(3))$ & 2.45 & $2.33(2.268(4))$ & 2.30 \\
$\mathrm{~d}_{\mathrm{M}-\mathrm{O}_{1}}$ & $1.88(1.837(2))$ & 1.91 & $2.03(1.962(2))$ & 2.06 & $2.00(1.956(4))$ & 2.04 \\
$\mathrm{~d}_{\mathrm{M}-\mathrm{O}_{2}}$ & $1.89(1.829(2))$ & 1.91 & $2.03(1.965(2))$ & 2.06 & $2.00(1.963(4))$ & 2.02 \\
$\mathrm{~d}_{\mathrm{M}-\mathrm{Cl}_{1}}$ & $2.30(2.323(1))$ & 2.27 & $2.44(2.441(1))$ & 2.41 & $2.38(2.409(2))$ & 2.36 \\
$\mathrm{~d}_{\mathrm{M}-\mathrm{Cl}_{2}}$ & $2.35(2.350(1))$ & 2.29 & $2.50(2.474(1))$ & 2.45 & $2.43(2.451(2))$ & 2.39 \\
$\mathrm{~d}_{\mathrm{M}-\mathrm{C}}$ & $2.22(2.184(3))$ & 2.24 & $2.40(2.358(3))$ & 2.41 & $2.33(2.333(6))$ & 2.35 \\
$\mathrm{~d}_{\mathrm{O}_{1}-\mathrm{O}_{2}}$ & 3.72 & 3.75 & 3.94 & 3.99 & 3.92 & 3.96 \\
$\mathrm{~d}_{\mathrm{O}_{m}-\mathrm{Cl}_{1}}$ & 4.58 & 4.49 & 4.91 & 4.83 & 4.71 & 4.64 \\
$\mathrm{~d}_{\mathrm{C}-\mathrm{Cl}_{2}}$ & 4.50 & 4.51 & 4.73 & 4.72 & 4.68 & 4.70 \\
\hline \hline
\end{tabular}

significantly the degree of distortion of the octahedral metal coordination. Overall, a feature that remains unaffected by the type of TM is the evolution of the structure of octahedral metallic site upon removal of one electron. We can then infer that the oxidation of the complex is something intrinsically related to the nature of the environment 5 surrounding and coordinating the TM rather than the chemistry of the TM center. This statement can be visualized in Figure 2-right, where these local octahedral conformation around the TM is shown in detail.

Finally it is worth mentioning that weak Metal - Hydrogen, van der Waals like interactions, at distances higher than the first neighbors, do not seem to play any significant 10 role in our complexes. On the one hand agostic interactions $[50,51]$ are ruled out on a simple geometric point of view, while anagostic interactions [52] that could have been considered are only found for M...H distances lower than 2.6-2.8 $\AA$ against at least $3.2 \AA$ in our models. 
Table 2: Bond angles (degrees) between the Metal atoms and their nearest neighbors. For each metal the columns read like: right= neutral system and left= charged system (see Figure 2 for details regarding the atomic labels). The uncertainty of the simulated values is $\pm 4^{\circ}$. The experimental values obtained by X-ray crystallography analysis are reported in parenthesis and taken from Refs. [22, 23, 25] for Ti, $\mathrm{Zr}$, and $\mathrm{Hf}$ complexes, respectively.

\begin{tabular}{lrrrrrr}
\hline \hline & \multicolumn{1}{c}{$\mathrm{Ti}$} & $\mathrm{Zr}$ & \multicolumn{1}{c}{$\mathrm{Hf}$} \\
& \multicolumn{1}{c}{0} & \multicolumn{1}{c}{0} & \multicolumn{1}{c}{0} & \multicolumn{1}{c}{+1} \\
\hline $\mathrm{O}_{1}-\mathrm{M}-\mathrm{O}_{2}$ & $161.7(159.19(9))$ & 158.6 & $152.8(153.26(8))$ & 152.0 & $156.4(154.7(2))$ & 154.0 \\
$\mathrm{O}_{m}-\mathrm{M}-\mathrm{O}_{1}$ & 85.5 & 83.1 & 82.6 & 82.7 & 82.8 & 82.1 \\
$\mathrm{O}_{m}-\mathrm{M}-\mathrm{O}_{2}$ & 85.4 & 84.1 & 83.6 & 83.2 & 83.5 & 83.6 \\
$\mathrm{O}_{m}-\mathrm{M}-\mathrm{Cl}_{1}$ & 172.7 & 169.6 & 172.5 & 167.8 & $174.0(179.1(1))$ & 171.0 \\
$\mathrm{O}_{m}-\mathrm{M}-\mathrm{Cl}_{2}$ & 83.5 & 88.4 & 78.9 & 81.1 & 82.4 & 85.5 \\
$\mathrm{O}_{m}-\mathrm{M}-\mathrm{C}$ & $77.6(88.49(3))$ & 82.1 & 70.7 & 72.0 & 75.8 & 80.2 \\
$\mathrm{C}_{-} \mathrm{M}-\mathrm{Cl}_{1}$ & 95.1 & 87.5 & 101.8 & 95.8 & $98.2(96.8(1))$ & 90.9 \\
$\mathrm{C}-\mathrm{M}-\mathrm{Cl}_{2}$ & 161.1 & 170.5 & 149.7 & 153.1 & $158.2(166.2(1))$ & 165.7 \\
$\mathrm{C}-\mathrm{M}-\mathrm{O}_{1}$ & $81.3(81.77(10))$ & 80.9 & $76.7(76.4(1))$ & 76.3 & 79.0 & 78.0 \\
$\mathrm{C}-\mathrm{M}-\mathrm{O}_{2}$ & 81.3 & 80.3 & $76.4(77.3(1))$ & 76.4 & 79.3 & 78.3 \\
$\mathrm{Cl}_{1}-\mathrm{M}-\mathrm{Cl}_{2}$ & $103.8(97.71(4))$ & 102.0 & 108.5 & 111.2 & 103.6 & 103.4 \\
$\mathrm{Cl}_{1}-\mathrm{M}-\mathrm{O}_{1}$ & 93.3 & 95.1 & 95.2 & 94.4 & 95.7 & 94.8 \\
$\mathrm{Cl}_{1}-\mathrm{M}-\mathrm{O}_{2}$ & 93.7 & 94.4 & 95.4 & 94.3 & 96.0 & 96.0 \\
$\mathrm{Cl}_{2}-\mathrm{M}-\mathrm{O}_{1}$ & 97.6 & 97.9 & 100.8 & 101.1 & 98.5 & 99.9 \\
$\mathrm{Cl}_{2}-\mathrm{M}-\mathrm{O}_{2}$ & 97.2 & 98.9 & 99.4 & 100.5 & 98.6 & 100.5 \\
\hline \hline
\end{tabular}

\subsection{Electronic properties}

The band gap for the M-carbene complexes are summarized in table 3 and KohnSham energy levels are sketched in in Figure 3 and 4, where $\alpha$ and $\beta$ refer to the up and down spin, respectively and consistently with the spin-unrestricted approach adopted.

5 By looking at these Figures, the positively charged complex obtained upon removal of one electron carries a singly occupied highest molecular orbital (SOMO hereafter) with the electron subtracted from the $\alpha$ spin component. Hence, the results in table 3 mostly reflect the fact that only the gap of this $\alpha$ component is affected, and reduces significantly by $\simeq 1.7$ up to $\simeq 2.3 \mathrm{eV}$ with respect to the neutral system.

Table 3: SOMO-LUMO band gap in $\mathrm{eV}$ for the studied organometallic complexes, and $\delta_{\mathrm{SOMO}}(\alpha-\beta)$ for the +1 systems. For each TM the columns read like: left= neutral system, right= charged system.

\begin{tabular}{lcccccc}
\hline \hline & \multicolumn{2}{c}{ Ti } & \multicolumn{2}{c}{$\mathrm{Zr}$} & \multicolumn{2}{c}{ Hf } \\
& 0 & +1 & 0 & +1 & 0 & +1 \\
\hline$\alpha$ & 2.046 & 0.307 & 2.265 & 0.311 & 2.656 & 0.373 \\
$\beta$ & 2.046 & 2.141 & 2.265 & 2.354 & 2.656 & 2.669 \\
$\delta_{\text {SOMO }}(\alpha-\beta)$ & & -0.01 & & -0.05 & & -0.06 \\
\hline \hline
\end{tabular}

10 As mentioned in the former paragraph, on the basis of the stability of the molecular structures examined, we focus on the most stable conformation of the complexes (figure 1-a). To get a better insight into the amount of localization of the orbitals contributing to the band structure discussed above, each Kohn-Sham state is projected onto atomic orbitals centered on the atoms. This turns out to be of particular interest for the 

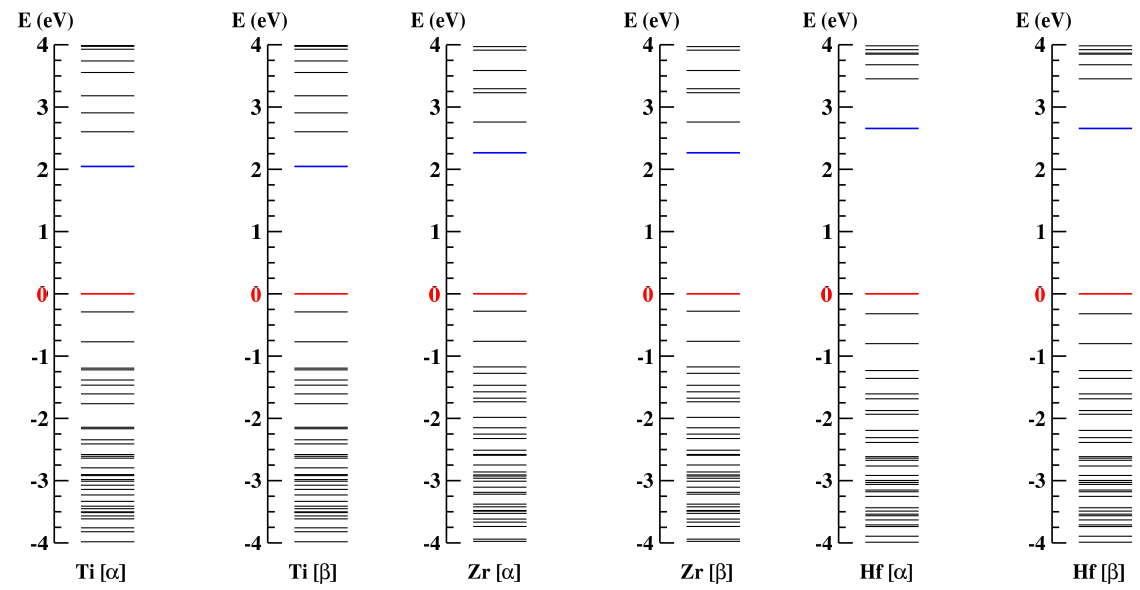

Figure 3: (Color online) Band diagrams for the neutral complexes, from left to right: $\mathrm{Ti}, \mathrm{Zr}$ and Hf. The zero in energy is placed at the SOMO level and highlighted in red. The SUMO level is highlighted in blue.

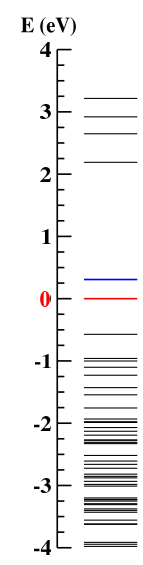

Ti $[\alpha]$
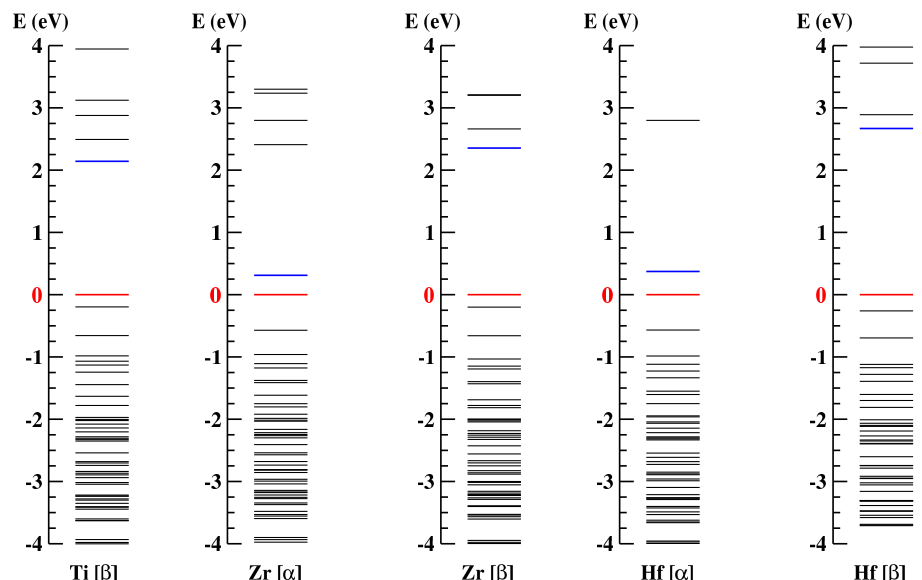

Figure 4: (Color online) Band diagrams for the charged (+1) complexes, from left to right: $\mathrm{Ti}, \mathrm{Zr}$ and $\mathrm{Hf}$ The zero in energy is placed at the SOMO level and highlighted in red. The SUMO level is highlighted in blue.

eigenstates around the band gap, since these the ones mainly involved in chemical reactions. As in any molecular system, the energetic picture provided by the KS electronic levels has to be complemented by the associated one-electron density $\left|\phi_{i}(\mathbf{x})\right|^{2}$, especially for the SOMO and its unoccupied counterpart, the singly unoccupied molecular 5 orbital (SUMO) of the energetically lowest empty band. Since the results were identical independently of the TM center, we report only one set of data, specifically those for the Zirconium based system. Furthermore, and according to the results shown in the band diagrams, in Figure 5 only the $\beta$ molecular orbitals ( $\beta-\mathrm{MO}$ ) are shown for the neutral system, $\alpha$ MO being identical.

Analogously, only the $\beta$-MO are shown for charged complex. This provides at glance a comparison between the neutral and the charged system in which one electrons is missing in the $\alpha$ spin component. By looking at the electronic states reported in Fig- 
$\mathrm{SUMO}+1$
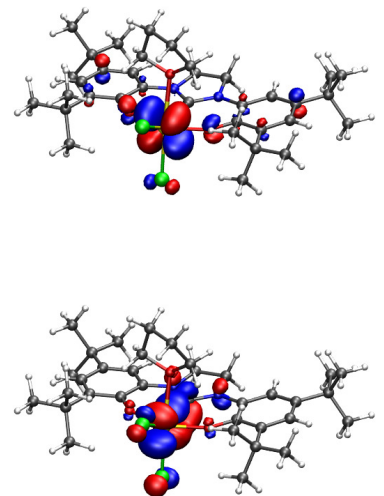

SUMO

SOMO
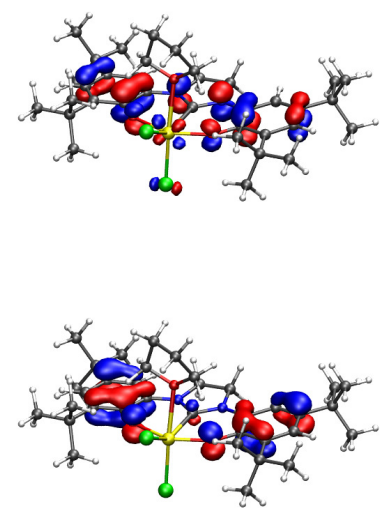
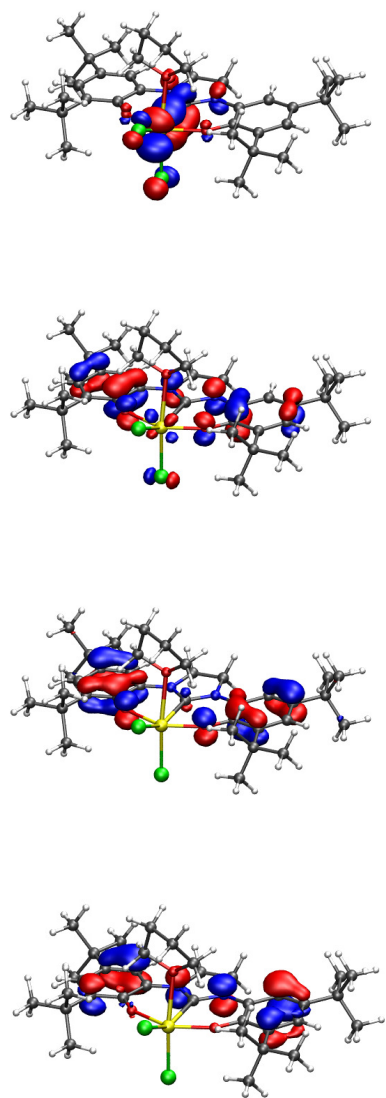

SOMO - 1

Figure 5: (Color online) Probability isodensity surfaces, taken at the value $0.05 \mathrm{eV} / \mathrm{a} . \mathrm{u} .^{3}$, for the eigenstates near the Single highest Occupied (SOMO) and Single lowest Unoccupied (SUMO) $\alpha$ molecular orbitals: a) on the left side: for the neutral $\mathrm{Zr}$ complex, b) and on the right side: for the charged $\mathrm{Zr}$ complex.

ure 5, from the SOMO-1 to the SUMO+1, no particular change is observed between the neutral and the charged complex. The SOMO-1 orbitals result to be delocalized over the carbene ligand, while the SOMO states are mostly delocalized over the carbene molecule with some contribution from the $d_{x y}$ orbital of the TM center and $p$ orbitals of the $\mathrm{Cl}_{1}$ atoms (see Figure 2 for the atomic labeling). This is not unexpected and reflects what has been observed in metal-NHC complexes in general.[53, 54] Indeed, in the neutral complex the electron to be removed can be more easily extracted from the carbene ligand, since the metal center is generally in a cationic state and, as such, already deprived of valence electrons. Because of the $\mathrm{Cl}$ atoms directly coordinated to the TM and the two $\mathrm{O}$ atoms of the carbene, the metal centers have to forfeit their valence electrons to the chemical bonds with the ligand becoming cationic $M(+I V)$, thus making virtually impossible to extract electron(s) from the deeper levels of the TM center. The SUMO and SUMO+1 MO are mostly contributions arising from the $d_{x y}(\mathrm{SUMO}+1)$ and $d_{y z}$ orbitals (SUMO) of the metal cation, with minor lobes coming 15 from the $\mathrm{Cl}_{1}, \mathrm{O}_{1}, \mathrm{O}_{2}$ atoms, and the carbene ligand.

The strength of the carbene-metal bond depends both on electrostatics and on orbital interactions, which are both determined by the electronic properties of the carbene 
as well the metal. The $\sigma$-donor and $\pi$-acceptor capabilities of different carbenes correlate with the energy of the $\sigma$-symmetric lone pair orbital the relevant $\sigma^{*}$-orbital (often the LUMO) for overlap with the metal's d-orbitals. [20] For instance, the decomposition of the $\sigma-$ and $\pi$-bonding interactions for the $[\mathrm{NHC}] \mathrm{TiCl}_{4}$ complex, was found 5 to show a carbene-metal bond made of $87 \%$ of $\sigma$-bonding (NHC to $\mathrm{M}$ ) and $13 \%$ of $\pi$-bonding (M to NHC). $[55,20]$ The degree of $\sigma$-donor and $\pi$-acceptor capabilities depends on the type of ligand (e.g. saturated vs unsaturated NHC) as well as the proton affinity and basicity of the carbene. The saturated NHC is predicted to be both a stronger $\sigma$-donor and $\pi$-acceptor than the unsaturated NHC.

10 Figure 6 presents the $\alpha$ SUMO for the 3 complexes (Ti, Zr and Hf) in the neutral state and illustrates that the electronic structure of these M-carbene systems is consistent with a $\pi$ backbonding interaction between the metal's $d$ and the ligand $p$ orbitals.
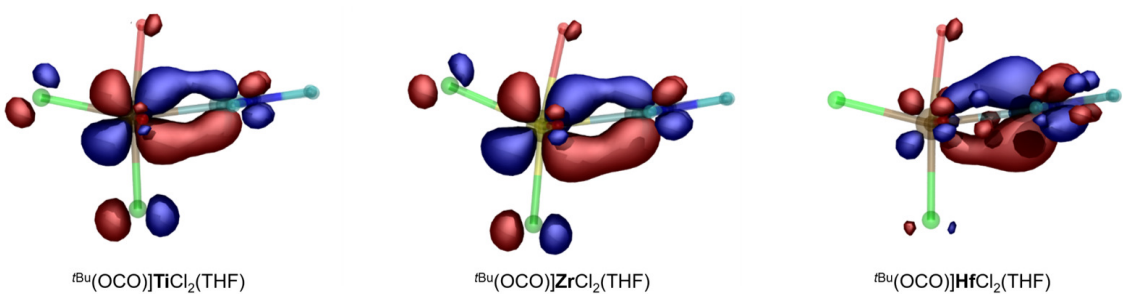

Figure 6: (Color online) Projection on the $\mathrm{O}_{m}-\mathrm{M}-\mathrm{C}$ plane of the eigenstates for the Single lowest Unoccupied (SUMO) $\alpha$ Molecular Orbitals for the neutral systems (probability isodensity surfaces taken at the value 0.05 eV/a.u. ${ }^{3}$ ). From left to right for the $\mathrm{Ti}, \mathrm{Zr}$ and $\mathrm{Hf}$ complexes, the representation is centered around the metal. Some atoms from the THF and the carbene ligand are hidden to clarify the visualization.

In Figure 8 the spin density distribution of the charged complex provides further sup15 port to the picture of a delocalization of the single electron on the carbene molecule. On a geometrical standpoint, the structure of the carbene ligand is not deeply affected by the removal of one electron. This is reflected in the evolution of its planarity according on the charge of the complex (see table 4), quantified by measuring the angles between the planes defined by each ring structure in the molecule, as sketched in Figure 7.

Table 4: Angles (degrees) between the planes defined by the aromatic cycles in the carbene ligand in figure 7. For each metal the columns read like: left $=0=$ neutral system and right $=+1=$ charged system.

\begin{tabular}{ccccccr}
\hline \hline & \multicolumn{2}{c}{ Ti } & \multicolumn{2}{c}{ Zr } & \multicolumn{2}{c}{ Hf } \\
& & & & & & \\
& 0 & +1 & 0 & +1 & 0 & +1 \\
\hline $\mathbf{1 - 3}$ & 0.70 & 5.29 & 0.35 & 0.17 & 0.25 & 4.01 \\
$\mathbf{1 - 2}$ & 7.33 & 2.88 & 4.09 & 3.32 & 5.11 & 11.23 \\
$\mathbf{2 - 3}$ & 7.44 & 8.56 & 3.99 & 3.22 & 4.27 & 7.28 \\
\hline \hline
\end{tabular}

Results in table 4 highlight that the evolution of the geometry of the carbene ligand with the charge is not as simple as the octahedral symmetry of the TM site. Specifically, the type of changes that the carbene can undergo depend on the nature of the metallic ion (see table 4). More precisely, for Ti the bending of the carbene molecule slightly increases upon electron removal, slightly decreases for $\mathrm{Zr}$, and increases sig- 


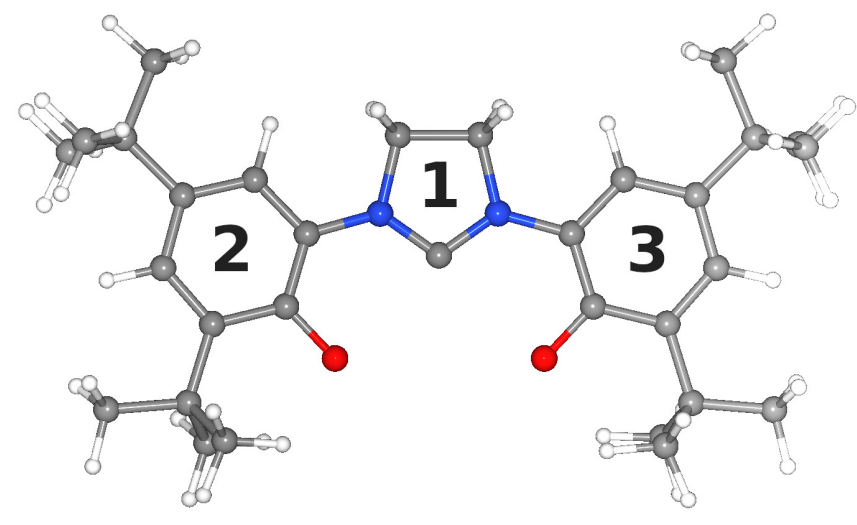

Figure 7: (Color online) Schematic of carbene molecule linked to the metal, the THF molecule is at the viewer position.

nificantly for Hf. 

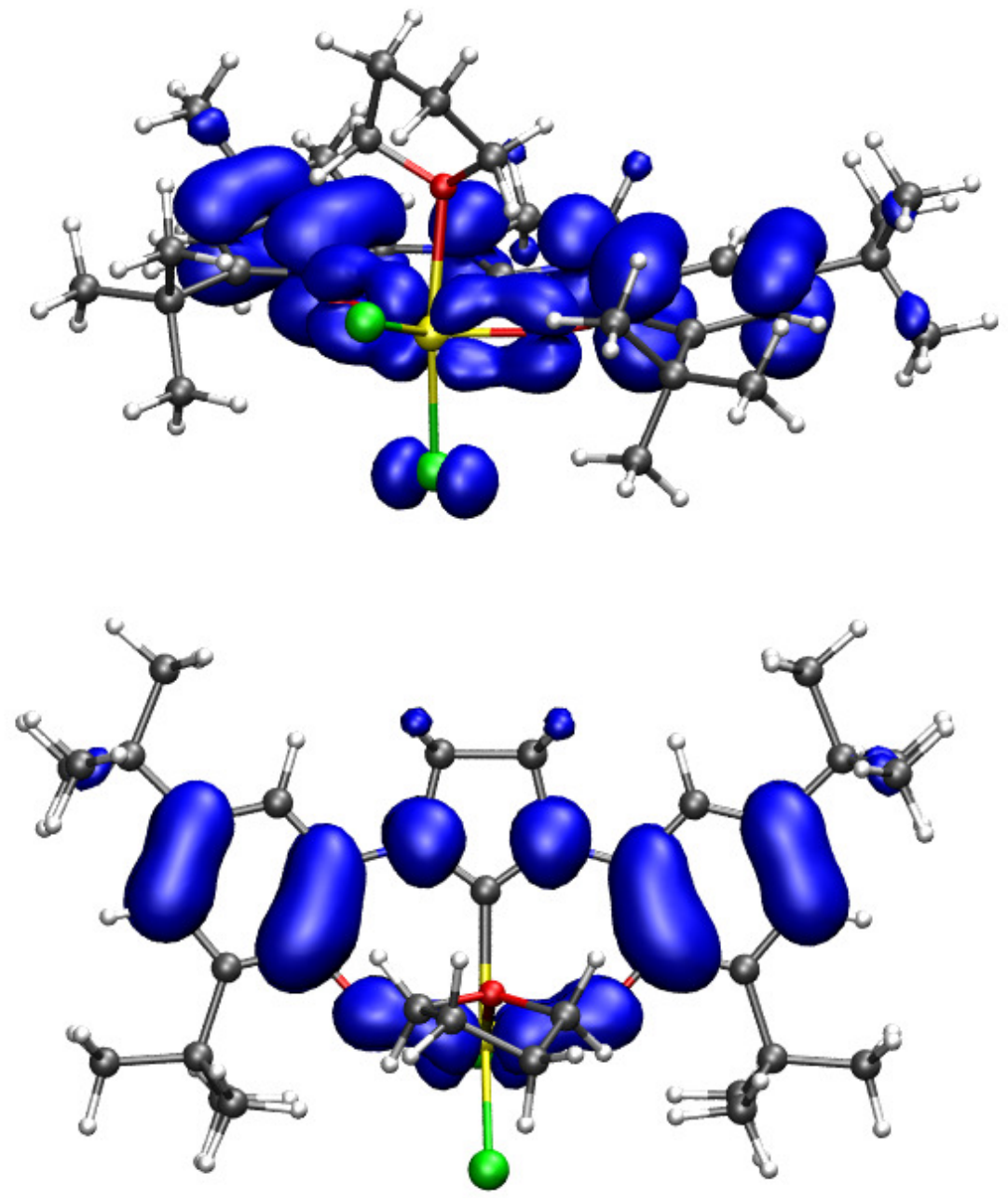

Figure 8: (Color online) The probability isodensity surfaces, taken at the value $-0.001 \mathrm{eV} / \mathrm{a} . \mathrm{u} .{ }^{3}$, for the spin density of the charged $\mathrm{Zr}$ complex. 


\subsection{Bader charges analysis}

To quantify the amount of charge localized in proximity of the atomic sites and to attribute partial electron densities to each atomic environment, we resorted to the Bader analysis.[48, 49] This approach allows to encompass the problem of partitioning in an unbiased way the DFT total charge density distribution and to assign a numerical value to a specific site. We remind that in molecular systems like the ones we are focusing on, the charge density reaches a minimum between atoms (or a maximum in fully covalent bonds) and this is the selected distance to separate atomic contributions according Bader's procedure. The result of our analysis is summarized in table 5 and - Figure 9. In our analysis we computed also the quantity $\delta_{B v}=$ Bader charge - valence intended as the difference between the amount of charge computed by the Bader's analysis with respect to the valence of the isolated atom as used in the construction of the corresponding pseudopotential. Three-dimensional representations of the charged $\mathrm{Zr}$-Carbene complex $(+1)$ are shown in figure 9 to illustrate the results of this calcula-

15 tion. A comparison is presented between the usual representation using the chemical species as color map (Fig. 9-a), and representations using different color maps with respectively the Bader charges (Fig. 9-b), the $\delta_{B v}(+1)$ for the charged complex (Fig. 9-c), and the difference $\delta_{+1-0}=\delta_{B v}(+1)-\delta_{B v}(0)$ (Fig. 9-d). As expected Figure 9-a) and Figure 9-b) are quite similar. Fig. 9-c) shows that that the charge distribution is rather

20 homogeneous and close to zero (meaning neutral) over all the carbene molecule, the atoms that carry a charge, positive or negative, significant enough to be highlighted by the color map, being all located around the metallic site. Fig. 9-d) confirms that the electron is removed from the carbene molecule as suggested by the SOMO orbital of the neutral system (see Figure 5 left side) delocalized over the carbene ligand. The TM 25 center is positively charged, as expected for a cation, with a charge between $\simeq 2$ and $\simeq 4$. The $\mathrm{C}$ atom of the carbene molecule also caries a positive charge $\simeq 2$. Most of the negative charges appear to delocalized over the oxygen atom of the THF molecule, $\mathrm{O}_{m}$, and even more significantly on the oxygen, $\mathrm{O}_{1}$ and $\mathrm{O}_{2}$, and the $\mathrm{N}$ atoms of the carbene ligand (see Tab. 5 and Figure 9-c). From the octahedral metallic site point of 30 view, the removal of one electron does not significantly affect the charge distribution (see Tab. 5), as visually illustrated by Figure 9-d).

Table 5: Bader charges [49] around the metallic site in the octahedral conformation (see Figure 2 for details regarding the atomic labels). For each metal the columns read like: left= neutral system and right $=$ charged system, with $\delta_{B v}=$ Bader charge - valence. The difference between the $\delta_{B v}$, for the charged and the neutral systems, $\boldsymbol{\delta}_{+1-0}$, is also presented (bold font).

\begin{tabular}{|c|c|c|c|c|c|c|c|c|c|c|c|c|c|c|c|}
\hline & \multicolumn{5}{|c|}{$\mathrm{Ti}$} & \multicolumn{5}{|c|}{$\mathrm{Zr}$} & \multicolumn{5}{|c|}{$\mathrm{Hf}$} \\
\hline & 0 & $\delta_{B v}(0)$ & +1 & $\delta_{B v}(+1)$ & $\delta_{+1-0}$ & 0 & $\delta_{B v}(0)$ & +1 & $\delta_{B v}(+1)$ & $\delta_{+1-0}$ & 0 & $\delta_{B v}(0)$ & +1 & $\delta_{B v}(+1)$ & $\delta_{+1-0}$ \\
\hline $\mathrm{M}$ & 9.91 & +2.09 & 9.88 & +2.12 & +0.03 & 9.86 & +2.14 & 9.86 & +2.14 & $\mathbf{0}$ & 0.29 & +3.71 & 0.26 & +3.74 & -0.03 \\
\hline $\mathrm{O}_{m}$ & 7.10 & -1.10 & 7.12 & -1.12 & -0.02 & 7.12 & -1.12 & 7.02 & -1.02 & -0.10 & 7.16 & -1.16 & 7.20 & -1.20 & -0.04 \\
\hline $\mathrm{O}_{1}$ & 7.58 & -1.58 & 7.58 & -1.58 & 0 & 7.54 & -1.54 & 7.61 & -1.61 & -0.07 & 7.90 & -1.90 & 7.87 & -1.87 & +0.03 \\
\hline $\mathrm{O}_{2}$ & 7.60 & -1.60 & 7.62 & -1.62 & -0.02 & 7.54 & -1.54 & 7.62 & -1.62 & -0.08 & 7.87 & -1.87 & 7.86 & -1.86 & +0.01 \\
\hline $\mathrm{Cl}_{1}$ & 7.52 & -0.52 & 7.45 & -0.45 & $+\mathbf{0 . 0 7}$ & 7.52 & -0.52 & 7.52 & -0.52 & 0 & 7.88 & -0.88 & 7.80 & -0.80 & +0.08 \\
\hline $\mathrm{Cl}_{2}$ & 7.56 & -0.56 & 7.52 & -0.52 & +0.04 & 7.57 & -0.57 & 7.46 & -0.46 & $+\mathbf{0 . 0 9}$ & 7.83 & -0.83 & 7.84 & -0.84 & -0.01 \\
\hline $\mathrm{C}$ & 1.97 & +2.03 & 2.01 & +1.99 & +0.04 & 1.89 & +2.11 & 1.93 & +2.07 & +0.04 & 2.14 & +1.86 & 2.16 & +1.84 & -0.02 \\
\hline
\end{tabular}


a)
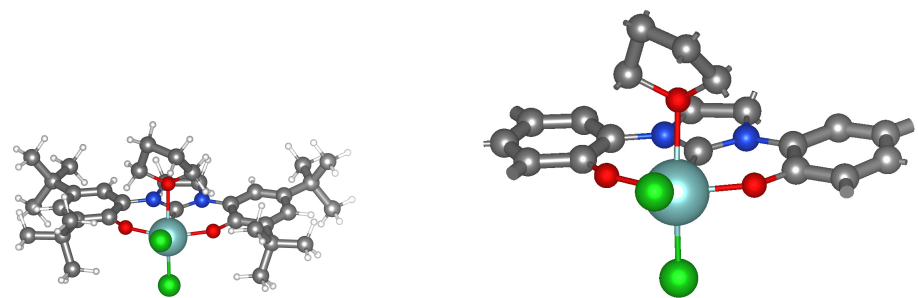

b)
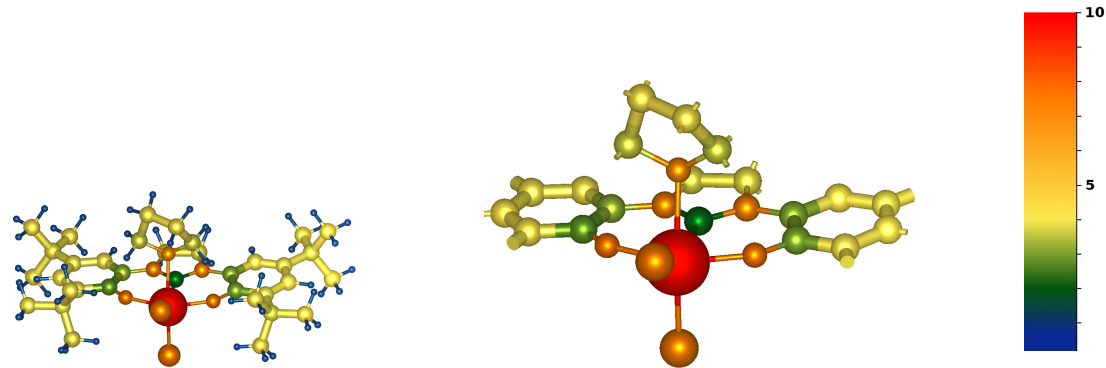

c)
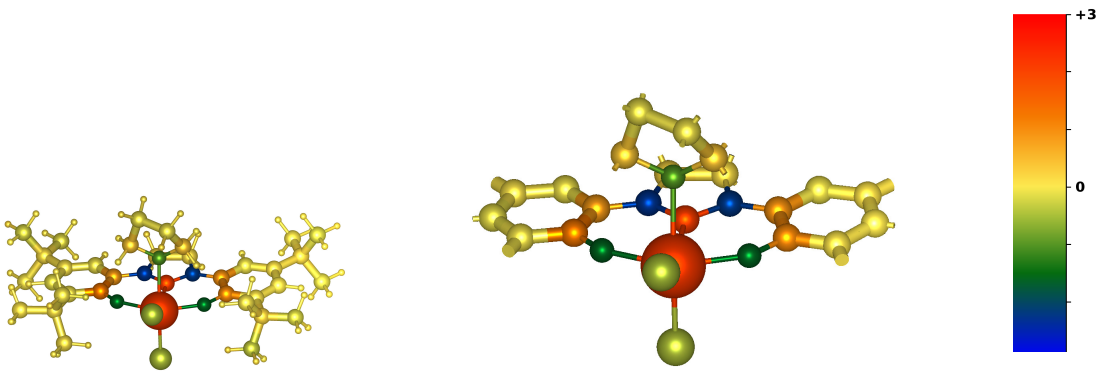

d)
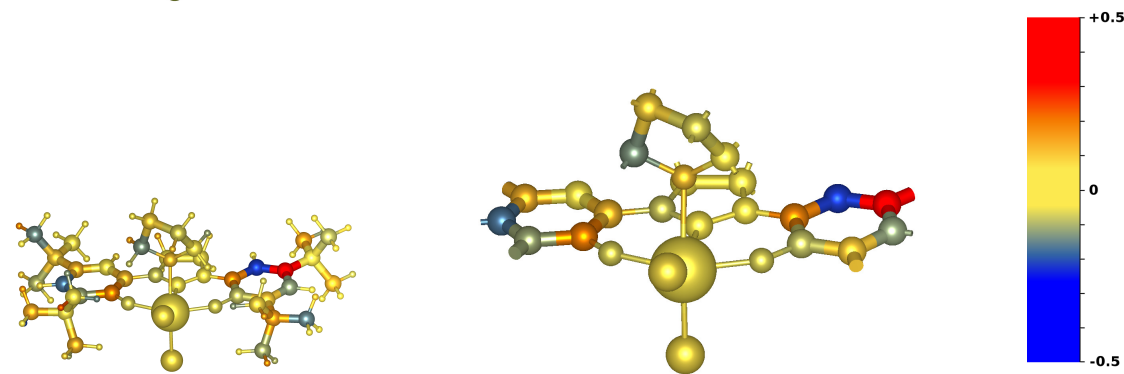

Figure 9: (Color online) Comparison of 3D representations for the charged Zr-Carbene complex, from top to bottom: a) standard color map using the atomic species, b) Bader charges, c) $\delta_{B v}(+1)$ (Bader charges valences) for the charged complex, and d) $\delta_{+1-0}$ : the difference between $\delta$ for the charged and the neutral complex, are used as color map respectively. When needed the color/charge scale is presented on the right side. (left: entire system, right zoom in on the M region). 


\section{Conclusion}

Motivated by the experimental activities of our group, we inspected via first principles methods, within the density functional theory framework, structural and electronic properties of carbene complexes in which the metal center is either $\mathrm{Hf}$ or Ti of Zr. These 5 systems, widely used in various catalytic processes of fundamental and industrial importance, show close similarities in both their conformations and electronic structure in their neutral state, evidencing the marginal role of the nature of the metallic center in their physical and chemical properties. Conversely, upon reduction, the removal of one electron in the system become responsible for remarkable modifications in the con-

10 figuration assumed by the ligand, in the HOMO-LUMO gap and in the partial charge distribution on the atoms composing the complex. An accurate selection of the metallic center can then become a key ingredient to tune the catalytic properties of the complex in view of practical applications.

\section{Acknowledgment}

This work was granted access by GENCI (Grand Equipement National de Calcul Intensif) under allocation DARI 201?096941 to the HPC resources of CINES/IDRIS. We acknowledge the HPC Mesocenter at the University of Strasbourg funded by the Equipex Equip@Meso project (Programme Investissements d'Avenir) and the CPER Alsacalcul/Big Data.

20 Figures 1, 2 (left), and 9 were created using the Atomes software. [56]

\section{Data availability}

The data that supports the findings of this study are available within the article.

[1] D. Bourissou, O. Guerret, F. P. Gabbaï, G. Bertrand, Chem. Rev. 100 (1) (2000) 39-92. doi:10.1021/cr940472u.

25 [2] S. Nolan, N-Heterocyclic Carbenes in Synthesis, John Wiley \& Sons, Ltd, 2006.

[3] F. Glorius, N-Heterocyclic Carbenes in Transition Metal Catalysis, SpringerVerlag Berlin Heidelberg, 2007.

[4] L. Mercs, M. Albrecht, Chem. Soc. Rev. 39 (2010) 1903-1912. doi:10.1039/ B902238B.

so [5] S. Díez-González, N-Heterocyclic Carbenes, From Laboratories Curiosities to Efficient Synthetic Tools, The Royal Society of Chemistry, 2011.

[6] D. J. Nelson, S. P. Nolan, Chem. Soc. Rev. 42 (16) (2013) 6723-6753.

[7] M. Hopkinson, C. Richter, M. Richter, F. Richter, Nature 510 (7506) (2014) 485-496.

35 [8] D. Zhang, G. Zi, Chem. Soc. Rev. 44 (2015) 1898.

[9] H. Wanzlick, H. Schonherr, Angew. Chem. Int. Ed. Engl. 7 (1968) 141.

[10] K. Öfele, Organomet. Chem. 12 (1968) 42. 
[11] A. Igau, H. Grutzmacher, A. Baceireido, G. Bertrand, J. Am. Chem. Soc.110 (1988) 6463.

[12] A. I. Arduengo, M. Kline, J. Calabrese, F. Davidson, J. Am. Chem. Soc.113 (1991) 9704.

5 [13] G. Horrer, M. Krahfus, K. Lubitz, I. Krummenacher, H. Braunschweig, U. Radius, Eur. J. Inorg. Chem. 2020 (2020) 281.

[14] C. Romain, S. Bellemin-Laponnaz, S. Dagorne, Coord. Chem. Rev. 422 (2020) 213411.

[15] M. Niehues, G. Kehr, G. Erker, B. Wibbeling, R. Frohlich, H. Blacque, O. Berke, J. Organomet. Chem. 663 (2002) 192.

[16] H. Jacobsen, A. Correa, C. Costabile, L. Cavallo, Organometallics 691 (2006) 4350.

[17] S. Büschel, T. Bannenberg, C. G. Hrib, A. Glückner, P. G. Jones, M. Tamm, J. Organomet. Chem. 694 (7) (2009) 1244-1250.

15 [18] A. Glückner, A. M. Arif, R. D. Ernst, T. Bannenberg, G. C. Daniliuc, P. G. Jones, M. Tamm, Inorganica Chimica Acta 364 (1) (2010) 23-29.

[19] S. Bellemin-Laponnaz, S. Dagorne, Chem. Rev. 114 (2014) 8747.

[20] D. Munz, Organometallics 37 (2018) 275.

[21] S. Bellemin-Laponnaz, R. Welter, L. Brelot, S. Dagorne, J. Organomet. Chem. 694 (2009) 604.

[22] C. Romain, K. Miqueu, J. M. Sotiropoulos, S. Bellemin-Laponnaz, S. Dagorne, Angew. Chem. 122 (2010) 2244.

[23] C. Romain, L. Brelot, S. Bellemin-Laponnaz, S. Dagorne, Organometallics 29 (2010) 1191.

25 [24] C. Romain, B. Heinrich, S. Bellemin-Laponnaz, S. Dagorne, Chem. Commun. 48 (2012) 2213.

[25] S. Dagorne, S. Bellemin-Laponnaz, C. Romain, Organometallics 32 (2013) 2736.

[26] C. Romain, S. Choua, J.-P. Collin, M. Heinrich, C. Bailly, L. Karmazin-Brelot, S. Bellemin-Laponnaz, S. Dagorne, Inorg. Chem. 53 (14) (2014) 7371-7376.

${ }_{30}$ [27] C. F. Harris, M. B. Bayless, N. P. van Leest, Q. J. Bruch, B. N. Livesay, J. Bacsa, K. I. Hardcastle, M. P. Shores, B. de Bruin, J. D. Soper, Inorg. Chem. 56 (20) (2017) 12421-12435.

[28] L. Gravogl, F. W. Heinemann, D. Munz, K. Meyer, Inorg. Chem. 59 (8) (2020) $5632-5645$.

[29] O. R. Luca, R. H. Crabtree, Chem. Soc. Rev. 42 (2013) 1440-1459. doi:10. 1039/C2CS35228A. 
[30] C. F. Harris, M. B. Bayless, N. P. van Leest, Q. J. Bruch, B. N. Livesay, J. Bacsa, K. I. Hardcastle, M. P. Shores, B. de Bruin, J. D. Soper, Inorg. Chem. 56 (2017) 12421-12435. doi:10.1021/acs.inorgchem.7b01906.

[31] E. O. Karaca, M. Akkoç, E. Öz, S. Altin, V. Dorcet, T. Roisnel, N. Gürbüz, . Çelik.

5 [32] R. Fırıncı, M. E. Günay, N. Özdemir, M. Dinçer, J. Mol. Struct. 1146 (2017) 267-272. doi:10.1016/j.molstruc.2017.06.012.

[33] F. P. Malan, E. Singleton, P. H. van Rooyen, J. Conradie, M. Landman, J. Mol. Struct. 1147 (2017) 235-243. doi:10.1016/j.molstruc.2017.06.086.

[34] R. Fraser, P. H. van Rooyen, J. de Lange, I. Cukrowski, M. Landman, J. Organo. Chem. 840 (2017) 11-22. doi:10.1016/j.jorganchem.2017.03.047.

[35] In the present work we use the 'neutral' and 'charged' nomenclature to refer to the entire complexes respectively in their their neutral (singlet state) and charged $(+1$, doublet state) charged states. within the framework of density functional theory the physical quantity is the global electron density and the total charge of the system is the only non-ambiguous quantity. the lack of one electron si clearly identified by simply integrating the electron density over the whole simulation cell, and not on any particular atom or molecular fragment of the system.

[36] A. D. Becke, Phys. Rev. A38 (6) (1988) 3098-3100.

[37] C. Lee, W. Yang, R. G. Parr, Phys. Rev. B37 (2) (1988) 785-789.

20 [38] G. Stapper, M. Bernasconi, N. Nicoloso, M. Parrinello, Phys. Rev. B59 (1999) 797-810.

[39] N. Troullier, J. L. Martins, Phys. Rev. B43 (3) (1991) 1993-2006.

[40] R. N. Barnett, U. Landman, Phys. Rev. B48 (4) (1993) 2081-2097.

[41] H. Jacobsen, L. Cavallo, Chem. Phys. Chem. 13 (2) (2012) 562-569. doi: 10. 1002 /cphc. 201100705.

[42] S. Grimme, Chem. Phys. Chem. 13 (6) (2012) 1407-1409. doi:10.1002/cphc. 201200094.

[43] S. Grimme, J. Comp. Chem. 27 (2006) 1787-1799.

[44] J. Hutter, A. P. Luthi, M. Parrinello, Comp. Mat. Sci.2 (1993) 244.

3о [45] M. I. J. Probert, J. Comput. Phys. 191 (2003) 130.

[46] R. Car, M. Parrinello, Phys. Rev. Let.55 (22) (1985) 2471-2474.

[47] Copyright IBM Corp. (1990-2020), copyright MPI für Festkörperforschung Stuttgart (1997-2001).

[48] R. Bader, Atoms in Molecules: A Quantum Theory, Oxford University Press, New York, 1990.

[49] G. Henkelman, A. Arnaldsson, J. H., Comp. Mat. Sci.36 (3) (2006) 354. doi : $10.1016 / j . c o m m a t s c i .2005 .04 .010$. 
[50] D. Braga, F. Grepioni, E. Tedesco, K. Biradha, G. R. Desiraju, Organometallics 16 (9) (1997) 1846-1856. doi:10.1021/om9608364.

[51] G. von Frantzius, R. Streubel, K. Brandhorst, J. Grunenberg, Organometallics 25 (1) (2006) 118-121. doi:10.1021/om050489a.

5 [52] H. V. Huynh, L. R. Wong, P. S. Ng, Organometallics 27 (10) (2008) 2231-2237. doi:10.1021/om800004j.

[53] A. Amokrane, S. Klyatskaya, M. Boero, M. Ruben, J.-P. Bucher, ACS Nano 11 (11) (2017) 10750-10760.

[54] R. Barhoumi, A. Amokrane, S. Klyatskaya, M. Boero, M. Ruben, J.-P. Bucher, Nanoscale 11 (44) (2019) 21167-21179.

[55] R. Tonner, G. Heydenrych, G. Frenking, Chem. Asian J. 2 (2010) 1555.

[56] https://www.conectus.fr/en/atomes.

\section{Supplementary information}

Table 6: Total energy, in $\mathrm{keV}$, for the $\left[{ }^{t \mathrm{Bu}}(\mathrm{OCO})\right] \mathrm{MCl}_{2}$ (THF) complexes considered in the present study. For each transition metal the table presents the total energy of the neutral, $E_{0}$, and charged systems, $E_{+1}$, for conformation a).

\begin{tabular}{lcc}
\hline \hline Metal & $E_{0}(\mathrm{keV})$ & $E_{+1}(\mathrm{keV})$ \\
\hline $\mathrm{Ti}$ & -10.482771 & -10.476555 \\
$\mathrm{Zr}$ & -10.163583 & -10.157413 \\
$\mathrm{Hf}$ & -8.9870689 & -8.9820978 \\
\hline \hline
\end{tabular}

